\title{
"Management of Gingival Hyperpigmentation" - 2 case reports
}

\author{
Dr. Verdine Virginia Antony, Dr. Rahamathulla Khan \\ ${ }^{I}$ ( Department of Periodontics, Sirte University, Libya.) \\ 2 ( Department of Orthodontics, Sirte University, Libya.)
}

\begin{abstract}
Gingival hyperpigmentation is mostly caused by the physiologic deposition of melanin by melanocytes. Melanin pigmentation of the gingiva is completely benign and does not present a medical problem. In "Gummy Smile" Patients, melanin gingival hyperpigmentation causes an esthetic problem and physiologic disturbances. Many methods are now available for depigmentation of the gingival. This paper reports two cases of gingival hyperpigmentation managed by surgical method.
\end{abstract}

Key Words: Gingiva, Melanin, Esthetic, Depigmentation.

\section{Introduction}

Oral pigmentation is a discoloration of the oral mucosa or gingiva associated with several exogenous and endogenous factors. Oral pigmented lesions can have various etiologies, namely- drugs, heavy metals, genetics, endocrine disturbances, inflammation and smoking [1,2,3]. This type of oral pigmentation is mostly located in the anterior labial gingiva, affecting females more than males [4]. The colour of the gingiva varies among different individuals and it is thought to be associated with cutaneous pigmentation. It depends on the vascular supply of the gingiva, epithelial thickness, degree of keratinisation of the epithelium and the presence of pigmented cells[5].

Melanin pigmentation is caused by melanin granules in gingival tissue, which are produced in melanosomes of melanocytes. Melanocytes are primarily located in the basal and suprabasal cell layers of the epithelium [6,7]. The color of the oral melanin pigmentation may vary from light to dark brown or black, depending on the amount and distribution of melanin in the tissue [2]. The oral pigmentation is due to the activity of melanocytes rather than the number of melanocytes in the tissue [8]. This hyperpigmentation is seen as a genetic variation in some populations independent of their age and sex. Hence it is termed as physiological or racial gingival pigmentation [5].Although physiologic melanin pigmentation is not a medical problem; patients complain that their black gums are unesthetic. This problem is aggravated in patients with a "gummy smile" or excessive gingival display while smiling.

Gingival depigmentation is a periodontal plastic surgical procedure wherein the gingival hyperpigmentation is removed or reduced by various techniques. The foremost indication for depigmentation therapy is the demand by a person for improved esthetics.

Numerous procedures have been developed for depigmentation of the gingiva, such as gingivectomy (Bergamaschi et al,1993), gingivectomy with free gingival autografting (Tamizi \& Taheri, 1996), acellular dermal martrix allografts (Pontes et al, 2006), electrosurgery (Gnanaesekhar \& Al-Duwairi, 1998), cryosurgery (Yeh, 1998), abrasion with diamond bur (Bishop,1994), and various types of lasers (Stabholz et al , 2003) [9]. Each technique has its own advantages and disadvantages.

This paper reports two cases of gingival hyperpigmentation managed by surgical method that does not require sophisticated instruments or apparatus yet yields esthetically acceptable results along with patient's satisfaction.

\section{Case Reports}

A 20 year old female patient (Figure:1) and a 27 year old male patient (Figure:2) reported to the department for routine oral prophylaxis. On intraoral examination, diffused blackish pigmentation of gingival was seen which was more prominent in the upper anterior region in both the cases. Clinically the gingival demonstrated signs of inflammation like bleeding on probing and color changes in both the patients. The unesthetic gingival pigmentation was pointed out to the patients and they were made aware about the array of aesthetic treatment options available. The patients had also noticed the gingival pigmentation since few years and of their own will opted to undergo the depigmentation procedure.

Depigmentation procedures were planned after obtaining patients consent. The patients were given oral hygiene instructions and they underwent oral prophylaxis in phase I of periodontal therapy. Depigmentation procedure was scheduled after the inflammation resolved. The procedure was carried out from canine to canine region in the maxillary anterior region for both cases (Figure:3, Figure:4) using de-epithelialization technique after adequate local anesthesia. The uppermost layer of the gingiva was carefully scraped using No. 15 blade which was held parallel to the long axis of the teeth. (Figure:5) Minimum amount of force was used to avoid 
post-operative gingival pitting. Bleeding was controlled with a sterile gauze pressure pack. Surgical areas were covered with a periodontal pack and post-operative instructions were given. Antibiotics and analgesic were prescribed for the management of pain. After one week the pack was removed and the surgical area examined. The healing was uneventful and satisfactory No post-surgical complications were encountered. Patients were recalled on a weekly basis to observe healing. At the end of one month, re-epithelization was complete and healing was found to be satisfactory (Figure:6, Figure 7).

Patient had no complaints of post-operative pain or sensitivity. The gingiva appeared healthy and no repigmentation was observed.

\section{Discussion}

Gingival melanin pigmentation causes esthetic concerns, and cosmetic therapy is becoming important for patients suffering from this problem. Melanin pigmentation is frequently caused by melanin deposition by active melanocytes located mainly in the basal layer of the oral epithelium. Pigmentation can be removed for esthetic reasons. Different treatment modalities have been used for this purpose [4]. The selection of a technique for depigmentation of the gingival should be based on clinical experience, patient's affordability and individual preferences.

Scalpel surgical technique is highly recommended in consideration of the equipment constraints that may not be frequently available in clinics. However, scalpel surgery causes unpleasant bleeding during and after the procedure and it is necessary to cover the surgical site with periodontal dressing for 7-10 days. It is known that the healing period for scalpel wounds is faster than other techniques[9]. Though the initial results of depigmentation procedure are highly encouraging, repigmentation is a common problem. The mechanism of repigmentation is not understood, but according to the migration theory, active melanocytes from the adjacent pigmented tissues migrate to the treated areas, causing repigmentation[10].Studies have shown that full clinical baseline repigmentation takes about 1.5-3 years[10]. In the present cases no incidence of repigmentation was observed at the end of 6 months. These cases are being followed up to estimate further the extent and rate of pigmentation.

\section{Conclusion}

The depigmentation procedure was successful and the patient was satisfied with the result. Hence we conclude that depigmentation of hyperpigmented gingival by the scraping technique using the scalpel is one of the most economic and also does not require extensive armamentation.

However it is safe to conclude that the procedure adopted is quite simple, cost effective and less painful with minimal tissue loss and hence can be repeated without complication keeping in mind the fact that repigmentation is a possibility in most cases.

\section{References}

[1]. Dummet CO : First symposium on oral pigmentation. Journal of Periodontology, 1960; 31(5): 345-385.

[2]. Kauzman A, Pavone M, Blanas N, Bradley G. Pigmented lesions of the oral cavity: review, differential diagnosis, and case presentations. J Can Dent Assoc. 2004;70:682-683.[PubMed]

[3]. Meleti M, Vescovi P, Mooi WJ, van der Waal I. Pigmented lesions of the oral mucosa and perioral tissues: a flow-chart for the diagnosis and some recommendations for the management. Oral Surg Oral Med Oral Pathol Oral Radiol Endod. 2008;105:606 616.[PubMed]

[4]. Hedin CA, Pindborg JJ, Axéll T. Disappearance of smoker's melanosis after reducing smoking. J Oral Pathol Med. 1993;22:228230. [PubMed]

[5]. Almas K and Sadiq W. Surgical treatment of melanin pigmented gingiva: An esthetic approach. Indian journal of Dental Research 2002; 13(2): 70-73.

[6]. Rosa DS, Aranha AC, Eduardo Cde P, Aoki A. Esthetic treatment of gingival melanin hyperpigmentation with Er:YAG laser: shortterm clinical observations and patient follow-up. J Periodontol. 2007;78:2018-2025.[PubMed] .

[7]. Ozcelik O, Cenk Haytac M, Kunin A, Seydaoglu G. Improved wound healing by low-level laser irradiation after gingivectomy operations: a controlled clinical pilot study. J Clin Periodontol. 2008;35:250-254. [PubMed]

[8]. Roshna T, Nandakumar K. (2005). Anterior Esthetic gingival depigmentation and crownlenthening: Report of a case. J Contemp Dent Pract, (6)3;139-147.

[9]. Perlmutter S,Tal H. (1986).Repigmentation of the gingiva following surgical injury Periodontol, 57:48-50.

[10]. Begamaschi O,Kon S,Doine AI,Ruben MP.(1993). Melanin repigmentation after gingivectomy :A five year clinical and transmission Electron Microscopic Study in Humans.Int Journal of periodontic's and restorative Dentistry, 13(1):85-92. 


\section{LEGENDS FOR FIGURES}

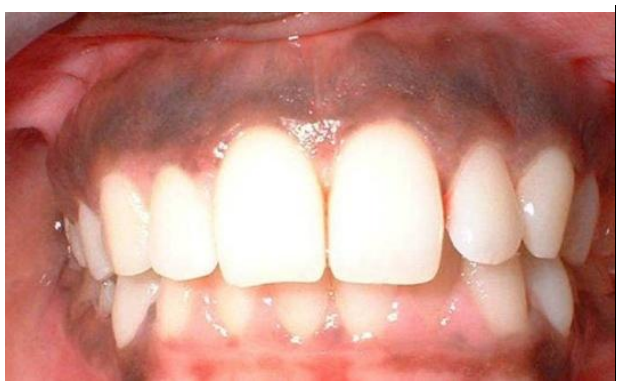

Figure 1:

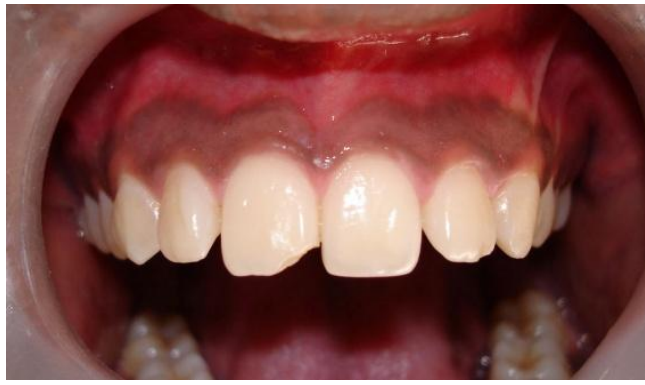

Figure 2:

Pre-op view showing highly pigmented upper labial gingiva

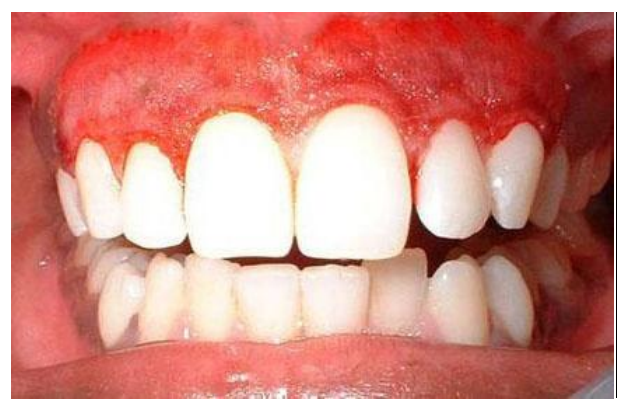

Figure 3:

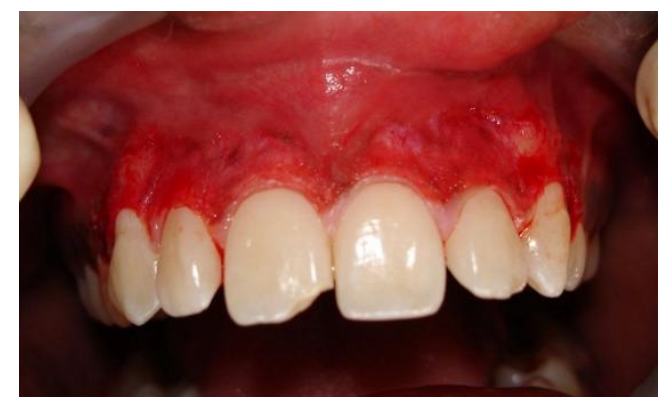

Figure 4:

Immediately after excising the epithelium

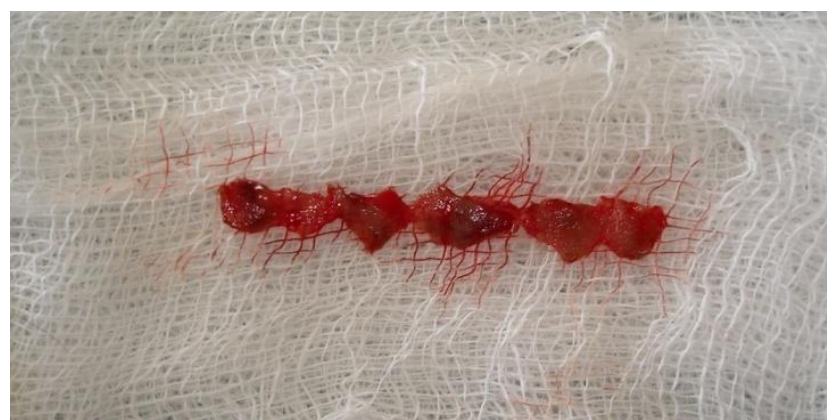

Figure 5: Excised epithelium

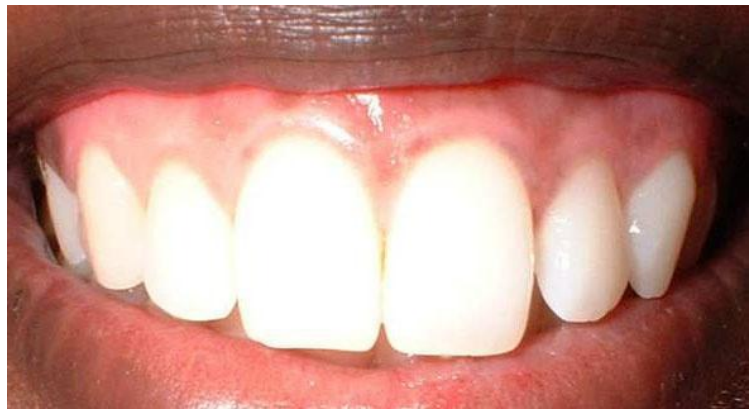

Figure 6:

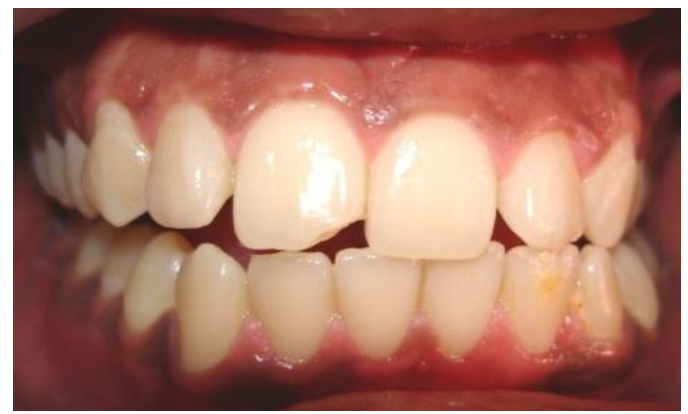

Figure 7:

Post-op 1 month. Depigmented gingiva that is pink \& healthy. 